\title{
Is It Possible to Predict the Outcome of IVF Treatment in Poor Response Patients?
}

\author{
Adi Uretzky1, Avraham Harlev1, Limor Man1, Eitan Lunenfeld1, Iris Har-Vardi1, \\ Michael Friger ${ }^{2}$, Eliahu Levitas ${ }^{1}$ \\ ${ }^{1}$ Fertility and IVF Unit, Department of Obstetrics and Gynecology, Soroka University Medical Center, \\ Beer-Sheva, Israel \\ ${ }^{2}$ Department of Epidemiology, Faculty of Health Sciences, Ben-Gurion University of the Negev, Beer-Sheva, Israel \\ Email: levitase@bgumail.bgu.ac.il
}

Received 16 June 2014; revised 15 July 2014; accepted 8 August 2014

Copyright (C) 2014 by authors and Scientific Research Publishing Inc.

This work is licensed under the Creative Commons Attribution International License (CC BY). http://creativecommons.org/licenses/by/4.0/

c) (i) Open Access

\begin{abstract}
Poor ovarian response (POR)-retrieval of 3 or fewer eggs, is a challenging issue in IVF. A retrospective study included POR women who underwent 386 IVF cycles. The data were classified in four groups according to women's age (years) during the treatment cycle: 1) 20 - $34(n=133), 2)$ 35 - $39(n=133), 3) 40-42(n=78), 4) 43-47$ y $(n=42)$, and correlated with the characteristics of the population. The clinical pregnancy rates for groups $1,2,3$, and 4 were: $23.3 \%, 12 \%, 2.6 \%$, $4.8 \%$, respectively. It was found to be significantly higher $(p<0.001)$ comparing group 1 with group 3 patients. The "take home baby" rate was much lower $(p<0.001)$ in group 3; there were no deliveries in group 4. Delivery rates for groups $1,2,3$, and 4 were: $19.5 \%, 10.5 \%, 1.3 \%$ and $0 \%$, respectively. Intraabdominal adhesions were more common $(p=0.005)$ as the cause of infertility in group 3 women compared to groups 2 and $1: 24.4 \%$ compared with $9 \%$ and $9.8 \%$, respectively. According to multivariate regression analysis, the parameters that negatively reflect on the pregnancy rate in POR women are intraabdominal adhesions, POR in the past, and increased age. We suggest encouraging young POR patients to pursue IVF treatments since the "take home baby" rates are reasonably good.
\end{abstract}

\section{Keywords}

IVF, Poor Ovarian Response, Failed IVF Treatments

\section{Introduction}

Poor ovarian response (POR) to stimulation is a challenging issue in the field of infertility. Poor responders have

How to cite this paper: Uretzky, A., Harlev, A., Man, L., Lunenfeld, E., Har-Vardi, I., Friger, M. and Levitas, E. (2014) Is It Possible to Predict the Outcome of IVF Treatment in Poor Response Patients? Open Journal of Obstetrics and Gynecology, 4, 703-709. http://dx.doi.org/10.4236/ojog.2014.412098 
a higher incidence of cycle cancellation, lower fertilization rate, and lower pregnancy and implantation rates [1]. Data from the ASRM/SART register show that $14.1 \%$ of initiated cycles are canceled, and it is assumed that at least $50 \%$ of those were poor responders [2].

Since POR was initially described, diverse criteria have been used to define it. The lack of a uniform definition of a poor response in the past, led in 2011 to the development of the "Bologna criteria" for poor ovarian response [3]. The definition represents the first realistic attempt by the scientific community to standardize the definition of POR in a simple and reproducible manner.

The minimal criteria needed to define POR include the presence of at least two of the following three features: 1) advanced maternal age or any other risk factor for POR, 2) a previous POR, and 3) an abnormal ovarian reserve test (ORT). The new consensus enables comparing between studies to evaluate the different risk factors for POR and the contribution of different interventions to the success rate of treatments.

The etiology of poor response to ovarian stimulation is indeterminate; there are many factors estimated to affect POR, but clearly the ovarian response is related to increasing age. Studies have confirmed that the IVF pregnancy rates diminish with increasing age [4]-[6]. The cutoff age above which the pregnancy rate starts to diminish significantly was found to be 36 years. In younger patients, the causes of the poor response are uncertain, but it represents a step toward depletion of the ovarian pool [7]. Short menstrual cycle length, single ovary, ovarian cystectomy, cigarette smoking, unexplained infertility, chemotherapy, and radiotherapy are well-established risk factors [8]. Other risk factors such as ovarian endometriomata [9] and uterine artery embolization [10] are still controversial.

Several endocrine and ultrasonographic markers are used to assess the ovarian reserve [11]-[13]. However, these tests are not accurate enough, and for many women poor ovarian response is discovered only during the first IVF attempt. Moreover, there is no consensus on the precise management of this group of patients. An extensive number of treatment protocols for poor responders have been proposed in the context of IVF, but there is no evidence that any of the specific treatment strategies is more effective than another [14] [15].

The aim of our study was to compare pregnancy and birth rates between different age groups of patients with POR. We explored the factors that might be related to reduced ovarian response to gonadotropin treatment, while defining the group of patients with improved prognosis to achieve pregnancy despite POR.

\section{Materials and Methods}

A retrospective cohort study was performed at the IVF Unit of the Soroka University Medical Center from January 1999 to December 2009 based on data from the medical records. The study was approved by the IRB committee and includes 386 IVF cycles with 3 or fewer oocytes retrieved from 277 women aged 18 - 47.

The following exclusion criteria were applied: age below 18 and over 47, women who didn't complete the IVF treatment for non-medical reasons, cycles canceled due to failure to supply a sperm specimen, cycles canceled due to azoospermia, women who had a poor response at the initial IVF treatment but in the following cycles had an improved response and produced more than 3 oocytes, patients who used surrogate uterus.

The patients were stratified to four groups according to their age during the treatment cycle: group I-20 - 34 years, group II—35 - 39 years, group III—40 - 42 years, and group IV—43 - 47 years. For every age group demographic, gynecologic, obstetric, infertility, and IVF parameters were compared. The following data were recorded: age, indication for IVF treatment, risk factors suspected for poor ovarian response, pregnancies and miscarriages in the past, biochemical measures, number of oocytes retrieved and fertilized in an IVF cycle, the quantity of gonadotropins consumed in the given cycle, and the treatment outcome-pregnancy and delivery. Late miscarriage was defined as miscarriage starting at 16 weeks of gestation and later; abdominal surgery was defined as an abdominal non-reproductive system intervention; gynecological surgery was defined as reproductive system intervention, not including caesarean section or curettage. Obstetric history including past pregnancies, deliveries, miscarriages, chemical pregnancies, ectopic pregnancies, and induced abortions was compared.

\section{Statistical Analysis}

Comparison between the age groups was performed by Kruskal-Wallis. Chi-square test was used for qualitative variables, and Student's t-test or ANOVA for quantitative variables. Multivariate logistical regression analysis was applied for non-dependent variables that were statistically or clinically significant. Relationship between the variables was also analyzed. The dependent variable was clinical pregnancy yes/no. Statistical analysis was 
performed using SPSS software version 18 (statistical package for social science version 1 for Windows). P value $<0.05$ was considered statistically significant.

\section{Results}

A total of 277 women undergoing 386 IVF cycles were included: group I-20 - 34 years $(n=133)$, group II -35 - 39 years $(n=133)$, group III $-40-42$ years $(n=78)$, and group IV $-43-47$ years $(n=42)$.

Inspecting the general and gynecological characteristics of the groups, the BMI was significantly higher in group 1 compared to group 4 (Table 1). The rate of smokers was highest in group 4, and lowest in group 3; however, the differences did not reach statistical significance. Previous abdominal surgery was found to be more prevalent among younger women in group 1 compared to older patients in groups 3 and 4 . Inversely, gynecologic surgery in the past was more common in the older age group versus younger women. Curettage in the past was significantly more frequent in groups 2 , 3, and 4 compared to group 1 (Table 1). Concerning obstetrical history, the clinical data wasn't significantly different between the age groups, probably due to the small number of pregnancies in the past.

While investigating the infertility etiology, a significant prevalence of pelvic adhesions was found in group 3 compared to the other study groups (Table 2). The FSH level on menstrual day 2 was significantly higher in groups 3 and 4 compared to groups 1 and 2. The highest prolactin level was observed in group 2 and TSH level was significantly elevated in group 4 (although both were within normal range). A history of POR was significantly higher in group 3. Differences for other parameters such as male factor or tubal occlusion did not reach statistical significance (Table 2).

As shown in Table 3, the most common ovarian stimulation protocol used in the youngest age group was the long GnRH agonist protocol and the most common ovarian stimulation protocol in group 4, the oldest age group, was the short GnRH agonist protocol (50\%). In group 3, the number of short and long protocols was almost equally divided. GnRH antagonist protocol was more frequently applied as the patient's age advanced (Table 3). In all age groups the ICSI method was more prevalent than insemination of oocytes, but without statistical significance. Additionally, the fertilization rate for all 3 available oocytes was much higher in group 1 compared with the other age groups, namely: $21.1 \%, 10.5 \%, 12.8 \%$, and $7.1 \%$ for groups $1,2,3$, and 4 , respectively. Nevertheless, the differences were not statistically significant. Finally, the quantity of gonadotropins implied was highest in group 4 (mean $4283.92 \pm 1408.022 \mathrm{IU}$ ) and the lowest in group 1 (mean $3762.86 \pm 1622.153 \mathrm{IU}$ ); however, with no statistical significance (Table 3).

Significantly higher clinical and pregnancy rates were found in group 1 in comparison with the other study groups. Furthermore, the "take home baby" rate was significantly higher in group 1 compared to the other groups; there were no deliveries in group 4. Chemical pregnancies were more common in the younger age groups: $2.3 \%, 2.3 \%, 1.3 \%$, and 0 for groups $1,2,3$, and 4, respectively. However, the differences were not statistically significant (Table 4).

Using a multiple logistic regression analysis model for confounders affecting the pregnancy rate such as previous miscarriages, pelvic adhesions, POR in the past, and maternal age were found to negatively affect the pregnancy rate (Table 5).

Table 1. General and gynecological characteristics of the study groups.

\begin{tabular}{|c|c|c|c|c|c|}
\hline & $\begin{array}{c}\text { Age group I } \\
20-34(n=133)\end{array}$ & $\begin{array}{c}\text { Age group II } \\
35-39(\mathrm{n}=133)\end{array}$ & $\begin{array}{l}\text { Age group III } \\
40-42(n=78)\end{array}$ & $\begin{array}{l}\text { Age group IV } \\
43-47(n=42)\end{array}$ & $P$ Value \\
\hline Age (years \pm STD) & $30.48 \pm 3.17$ & $36.95 \pm 1.33$ & $41.04 \pm 0.81$ & $43.98 \pm 1.04$ & \\
\hline BMI & $26.86 \pm 6.82$ & $25.61 \pm 5.42$ & $25.83 \pm 5.15$ & $22.73 \pm 4.24$ & 0.03 \\
\hline Smoking (\%) & 29.3 & 27.8 & 23.1 & 38.1 & 0.377 \\
\hline Abdominal surgery (\%) & 8.3 & 6 & 2.6 & 4.8 & 0.395 \\
\hline Gynecological surgery (\%) & 9 & 9.8 & 17.9 & 21.4 & 0.055 \\
\hline Cesarean section (\%) & 8.3 & 15.8 & 10.3 & 11.9 & 0.28 \\
\hline Curettage (\%) & 11.3 & 30.1 & 28.2 & 23.8 & 0.001 \\
\hline PID (\%) & 4.5 & 5.3 & 9 & 4.8 & 0.568 \\
\hline
\end{tabular}

BMI—Body Mass Index; PID—Pelvic Inflammatory Disease. 
Table 2. Infertility characteristics of the study groups.

\begin{tabular}{cccccc}
\hline & $\begin{array}{c}\text { Age group I } \\
20-34(\mathrm{n}=133)\end{array}$ & $\begin{array}{c}\text { Age group II } \\
35-39(\mathrm{n}=133)\end{array}$ & $\begin{array}{c}\text { Age group III } \\
40-42(\mathrm{n}=78)\end{array}$ & $\begin{array}{c}\text { Age group IV } \\
43-47(\mathrm{n}=42)\end{array}$ & P Value \\
\hline Unilateral tubal occlusion (\%) & 18 & 14.3 & 16.7 & 4.8 & 0.2 \\
Bilateral tubal occlusion (\%) & 13.5 & 12.8 & 25.6 & 21.4 & 0.054 \\
Pelvic adhesions (\%) & 13 & 9 & 24.4 & 9.5 & 0.005 \\
Male factor (\%) & 81 & 66.9 & 53.8 & 64.3 & 0.308 \\
Unexplained infertility (\%) & 28 & 17.3 & 20.5 & 23.8 & 0.778 \\
Endometriosis (\%) & 14 & 6.8 & 6.4 & 7.1 & 0.63 \\
Menstrual length (days \pm STD) & $36.46 \pm 30.36$ & $29.69 \pm 14.26$ & $28.17 \pm 2.03$ & $29.48 \pm 11.31$ & 0.026 \\
FSH (IU/ml \pm STD) & $8.998 \pm 7.046$ & $9.637 \pm 5.11$ & $10.938 \pm 6.201$ & $10.274 \pm 5.311$ & 0.006 \\
Prolactinng/ml & $16.57 \pm 8.667$ & $17.26 \pm 10.854$ & $13.69 \pm 7.683$ & $16.83 \pm 3.369$ & 0.028 \\
TSH (IU/ml \pm STD) & $2.21 \pm 1.242$ & $2 \pm 1.074$ & $2.33 \pm 0.921$ & $2.93 \pm 1.701$ & 0.002 \\
POR in the past \% & 30.8 & 43.6 & 53.8 & 38.1 & 0.016 \\
\hline
\end{tabular}

Table 3. IVF cycles characteristics of the study groups.

\begin{tabular}{|c|c|c|c|c|c|}
\hline & $\begin{array}{c}\text { Age group I } \\
20-34(\mathrm{n}=133)\end{array}$ & $\begin{array}{c}\text { Age group II } \\
35-39(n=133)\end{array}$ & $\begin{array}{c}\text { Age group III } \\
40-42(n=78)\end{array}$ & $\begin{array}{l}\text { Age group IV } \\
43-47(n=42)\end{array}$ & $P$ Value \\
\hline \multicolumn{6}{|l|}{$\begin{array}{l}\text { Protocol } \\
(\%)\end{array}$} \\
\hline Long & 73.7 & 56.4 & 38.5 & 23.8 & \\
\hline Short & 12.8 & 24.8 & 37.2 & 50 & 0.001 \\
\hline Antagonist & 13.5 & 18.8 & 24.4 & 26.2 & \\
\hline \multicolumn{6}{|l|}{$\begin{array}{l}\text { Micro-manipulation } \\
\text { (\%) }\end{array}$} \\
\hline Insemination & 34.6 & 24.1 & 30.8 & 23.8 & \\
\hline ICSI & 46.6 & 59.4 & 50 & 57.1 & 0.564 \\
\hline Both & 3 & 2.3 & 1.3 & 0 & \\
\hline E2Peak & $1009.35 \pm 549.49$ & $930.59 \pm 487.41$ & $988.41 \pm 493.83$ & $845.14 \pm 406.37$ & 0.13 \\
\hline $\begin{array}{l}\text { Oocytes retrieved } \\
\text { (\%) }\end{array}$ & & & & & 0.305 \\
\hline 0 & 4.5 & 5.3 & 6.4 & 4.8 & \\
\hline 1 & 22.6 & 18 & 30.8 & 26.2 & \\
\hline 2 & 27.1 & 33.8 & 26.9 & 31 & \\
\hline 3 & 45.9 & 42.9 & 35.9 & 38.1 & \\
\hline $\begin{array}{l}\text { Oocytes fertilized } \\
\text { (\%) }\end{array}$ & & & & & 0.365 \\
\hline 0 & 21.8 & 24.8 & 25.6 & 31 & \\
\hline 1 & 35.3 & 33.1 & 41 & 31 & \\
\hline 2 & 21.8 & 31.6 & 20.5 & 31 & \\
\hline 3 & 21.1 & 10.5 & 12.8 & 7.1 & \\
\hline $\begin{array}{l}\text { Transferred embryos } \\
\text { (\%) }\end{array}$ & & & & & 0.763 \\
\hline 0 & 27.1 & 30.1 & 26.9 & 31 & \\
\hline 1 & 36.8 & 38.3 & 47.4 & 40.5 & \\
\hline 2 & 30.1 & 27.8 & 16.7 & 23.8 & \\
\hline 3 & 6 & 3.8 & 9 & 4.8 & \\
\hline Gonadotropins IU & $3762.86 \pm 1622.15$ & $4103.77 \pm 1548.52$ & $3968.04 \pm 1422.42$ & $4283.93 \pm 1408.02$ & 0.171 \\
\hline
\end{tabular}


Table 4. IVF outcome of the study groups.

\begin{tabular}{|c|c|c|c|c|c|}
\hline & $\begin{array}{c}\text { Age group I } \\
20-34(\mathrm{n}=133)\end{array}$ & $\begin{array}{c}\text { Age group II } \\
35-39(n=133)\end{array}$ & $\begin{array}{l}\text { Age group III } \\
40-42(\mathrm{n}=78)\end{array}$ & $\begin{array}{c}\text { Age group IV } \\
43-47(n=42)\end{array}$ & $P$ Value \\
\hline Clinical pregnancy (\%) & 23.3 & 12 & 2.6 & 4.8 & $<0.001$ \\
\hline Chemical pregnancy (\%) & 2.3 & 2.3 & 1.3 & 0 & 0.755 \\
\hline Ectopic pregnancy (\%) & 0.8 & 0 & 0 & 0 & 0.592 \\
\hline Late miscarriage & 3 & 4.5 & 9 & 9.5 & 0.171 \\
\hline Trimester 1 miscarriage (\%) & 2.3 & 1.5 & 0 & 4.8 & 0.295 \\
\hline Artificial abortion (\%) & 0.8 & 0 & 0 & 0 & 0.592 \\
\hline Delivery (\%) & 19.5 & 10.5 & 1.3 & 0 & $<0.001$ \\
\hline
\end{tabular}

Table 5. Multiple logistic regression analysis of pregnancy rate related factors.

\begin{tabular}{cccc}
\hline Factor & Odds ratio & C.I. 95\% of OR & 0.118 \\
\hline Miscarriage & 0.333 & $0.084-1.322$ & 0.213 \\
Artificial abortion & 1.972 & $0.677-5.749$ & 0.003 \\
Pelvic Adhesions & 3.716 & $0.575-8.767$ & $0.318-0.863$ \\
POR in the past & 0.524 & $0.247-1.008$ & 0.053 \\
Age $20-34$ & 0.499 & $0.017-0.360$ \\
Age $35-39$ & 0.079 & 0.001 & $0.041-0.809$ \\
\hline
\end{tabular}

POR-Poor Ovarian Response.

\section{Discussion}

The most prominent finding of the study is the significant difference in IVF outcomes between older and younger patients regardless of the fact that the number of retrieved eggs was similar for all age groups. The absence of a widely agreed uniform definition for POR made the comparison with previous studies of POR difficult, resulting in debatable non-proven treatment regimens of POR [3]. The advantage of the current study is the use of patient selection according to the ESHRE consensus on the definition of "poor response" to ovarian stimulation known as the Bologna criteria [3].

No statistical significance in the aspect of number of oocytes retrieved, oocytes fertilized, or number of embryos transferred was observed. Although younger women's follicles were not observed to secrete more estrogen or to produce more oocytes, they do have more pregnancies. Additionally, the number of fertilized oocytes in the younger group was improved with greater chances of fertilization of all 3 oocytes available. The clinical pregnancy rate was significantly higher in the youngest age group and, accordingly, deliveries were also more prevalent in this group. The reduced fecundity related to age may be explained by a decline in egg quality expressed by a gradual rise in chromosomal aberrations in the oocytes and embryos related to ageing [16]. It had been found that aneuploidy rates rise as the ovarian reserve decline [17]-[19] and that younger age doesn't protect against the negative consequences of a decline in ovarian reserve [20]. We have been able to demonstrate an inverse correlation between age group and the outcome of clinical pregnancy, which is in contrast with a previous study [21], where all the women defined as POR had the same clinical pregnancy rate, unrelated to age. However, the lack of compatibility does not necessarily imply a contradiction in the findings, since the study populations were different. In our study, POR was defined as retrieval of 3 oocytes or fewer, whereas in the previous study [21], POR was strictly defined by the Bologna criteria. This couldn't have been done in our study since it was carried out retrospectively during the years that AFC and AMH weren't clinically used.

It is well established that the ovarian follicle pool decreases with age [1] [22] and consequently higher gonadotropin doses are needed to achieve controlled ovarian stimulation (COS). In fact, the ovarian stimulation can be viewed as a dynamic assessment of the resting ovarian follicular pool [7]. When POR is observed during COS, an early sign of ovarian ageing or decreased ovarian reserve is suspected. Excluding causes for premature ovarian failure in young women related with genetic abnormalities (fragile $\mathrm{X}$, turner syndrome, etc.), autoimmune 
disease, or enzyme deficiency [8], the results of this study emphasize the dissimilarity of oocyte conception prognosis in young age as compared to older age POR patients despite a common pathway of reduced ovarian follicular pool. The young patient with POR has a significantly greater chance to conceive and deliver. These results contradict a previous study addressing the same question [20]. PID is known to be a risk factor for poor ovarian response [23] [24]. Positivity to Chlamydia antibody testing had been found associated with a higher rate of poor ovarian response, a finding that can point out that a Chlamydia infection has a bad influence on ovarian function [25]. Pelvic adhesions are a known sequel of pelvic infection, and therefore found to be related to ovarian dysfunction [25]. In the current study, a higher rate of pelvic adhesions in the older group, and a strong (reverse) correlation between pelvic adhesions and the outcome of clinical pregnancies were noted in accordance with the previous observation of adverse PID sequelae and POR.

A statistically significant relationship between menstrual length and age was observed. The length of the menstrual cycle was shorter with older age. This finding is in accordance with the results of another study [26]. However, we doubt the clinical significance of menstrual length on poor ovarian response. It is important to point out that all the women who had amenorrhea longer than 6 months were defined as women who have a menstrual length of 180 days. This minimized the statistical significance, because most of the women (5 out of 6) were in group 1.

POR in the past treatment history was found to be significantly different between the age groups and negatively affected the clinical pregnancy rate according to the logistic regression analysis.

It should be noted that in the age group $43-47$, despite $4.8 \%$ clinical pregnancy rate, no deliveries were accomplished. This finding raises the question of whether it is justified to perform an IVF treatment in women known to be POR and aged 43 or older.

As mentioned, clinical pregnancy rate in the young patients group with POR in comparison to the older groups is significantly higher. We suggest encouraging young POR patients to pursue IVF treatments while the chances for pregnancy and "take home baby" rates are reasonably good. Obviously more and larger studies strictly following the Bologna criteria are necessary to confirm our findings.

\section{References}

[1] Saldeen, P., Källen, K. and Sundström, P. (2007) The Probability of Successful IVF Outcome after Poor Ovarian Response. Acta Obstetricia et Gynecologica Scandinavica, 86, 457-461. http://dx.doi.org/10.1080/00016340701194948

[2] Society for Assisted Reproductive Technology and the American Society for Reproductive Medicine (2007) Assisted Reproductive Technology in the United States: 2001 Results Generated from the American Society for Reproductive Medicine/Society for Assisted Reproductive Technology Registry. Fertility and Sterility, 87, 1253-1266. http://dx.doi.org/10.1016/j.fertnstert.2006.11.056

[3] Ferraretti, A.P., La Marca, A., Fauser, B.C., Tarlatis, B., Nargund, G., Gianaroli, L. and ESHRE Working Group on Poor Ovarian Response Definition (2011) ESHRE Consensus on the Definition of "Poor Response" to Ovarian Stimulation for in Vitro Fertilization: The Bologna Criteria. Human Reproduction, 26, 1616-1624. http://dx.doi.org/10.1093/humrep/der092

[4] Baker, V.L., Luke, B., Brown, M.B., Alvero, R., Frattarelli, J.L., Usadi, R., Grainger, D.A. and Armstrong, A.Y. (2010) Multivariate Analysis of Factors Affecting Probability of Pregnancy and Live Birth with in Vitro Fertilization: An Analysis of the Society for Assisted Reproductive Technology Clinic Outcomes Reporting System. Fertility and Sterility, 94, 1410-1416. http://dx.doi.org/10.1016/j.fertnstert.2009.07.986

[5] Speroff, L. (1994) The Effect of Aging on Fertility. Current Opinion in Obstetrics \& Gynecology, 6, 115-120. http://dx.doi.org/10.1097/00001703-199404000-00002

[6] Tan, S.L., Royston, P., Campbell, S., Jacobs, H.S., Betts, J., Mason, B. and Edwards, R.G. (1992) Cumulative Conception and Livebirth Rates after in-Vitro Fertilisation. The Lancet, 339, 1390-1394. http://dx.doi.org/10.1016/0140-6736(92)91205-M

[7] Beckers, N.G., Macklon, N.S., Eijkemans, M.J. and Fauser, B.C. (2002) Women with Regular Menstrual Cycles and a Poor Response to Ovarian Hyperstimulation for in Vitro Fertilization Exhibit Follicular Phase Characteristics Suggestive of Ovarian Aging. Fertility and Sterility, 78, 291-297. http://dx.doi.org/10.1016/S0015-0282(02)03227-2

[8] De Vos, M., Devroey, P. and Fauser, B.C. (2010) Primary Ovarian Insufficiency. The Lancet, 376, 911-921. http://dx.doi.org/10.1016/S0140-6736(10)60355-8

[9] Shah, D.K. (2013) Diminished Ovarian Reserve and Endometriosis: Insult upon Injury. Seminars in Reproductive Medicine, 31, 144-149. http://dx.doi.org/10.1055/s-0032-1333479 
[10] Kaump, G.R. and Spies, J.B. (2013) The Impact of Uterine Artery Embolization on Ovarian Function. Journal of Vascular and Interventional Radiology, 24, 459-467. http://dx.doi.org/10.1016/j.jvir.2012.12.002

[11] Celik, H., Bildircin, D., Guven, D., Cetinkaya, M.B., Alper, T. and Batuoglu, A.S. (2012) Random Anti-Mullerian Hormone Predicts Ovarian Response in Women with High Baseline Follicle-Stimulating Hormone Levels: Anti-Mullerian Hormone in Poor Responders in Assisted Reproductive Treatment. Journal of Assisted Reproduction and Genetics, 29, 797-802. http://dx.doi.org/10.1007/s10815-012-9794-y

[12] Hendriks, D.J., Mol, B.W., Bancsi, L.F., TeVelde, E.R. and Broekmans, F.J. (2005) Antral Follicle Count in the Prediction of Poor Ovarian Response and Pregnancy after in Vitro Fertilization: A Meta-Analysis and Comparison with Basal Follicle-Stimulating Hormone Level. Fertility and Sterility, 83, 291-301. http://dx.doi.org/10.1016/j.fertnstert.2004.10.011

[13] Ng, E.H., Tang, O.S. and Ho, P.C. (2000) The Significance of the Number of Antral Follicles Prior to Stimulation in Predicting Ovarian Responses in an IVF Programme. Human Reproduction, 15, 1937-1942. http://dx.doi.org/10.1093/humrep/15.9.1937

[14] Kyrou, D., Kolibianakis, E.M., Venetis, C.A., Papanikolaou, E.G., Bontis, J. and Tarlatzis, B.C. (2009) How to Improve the Probability of Pregnancy in Poor Responders Undergoing in Vitro Fertilization: A Systematic Review and Meta-Analysis. Fertility and Sterility, 91, 749-766. http://dx.doi.org/10.1016/j.fertnstert.2007.12.077

[15] Shanbhag, S., Aucott, L., Bhattacharya, S., Hamilton, M.A. and McTavish, A.R. (2007) Interventions for "Poor Responders" to Controlled Ovarian Hyperstimulation (COH) in in-Vitro Fertilisation (IVF). The Cochrane Database of Systematic Reviews, No. 1, Article ID: CD004379.

[16] TeVelde, E.R. and Pearson, P.L. (2002) The Variability of Female Reproductive Ageing. Human Reproduction Update, 8, 141-154. http://dx.doi.org/10.1093/humupd/8.2.141

[17] Nasseri, A., Mukherjee, T., Grifo, J.A., Noyes, N., Krey, L. and Copperman, A.B. (1999) Elevated Day 3 Serum Follicle Stimulating Hormone and/or Estradiol May Predict Fetal Aneuploidy. Fertility and Sterility, 71, 715-718. http://dx.doi.org/10.1016/S0015-0282(98)00525-1

[18] Trout, S.W. and Seifer, D.B. (2000) Do Women with Unexplained Recurrent Pregnancy Loss Have Higher Day 3 Serum FSH and Estradiol Values? Fertility and Sterility, 74, 335-337. http://dx.doi.org/10.1016/S0015-0282(00)00625-7

[19] van Montfrans, J.M., van Hooff, M.H., Martens, F. and Lambalk, C.B. (2002) Basal FSH, Estradiol and Inhibin B Concentrations in Women with a Previous Down's Syndrome Affected Pregnancy. Human Reproduction, 17, 44-47. http://dx.doi.org/10.1093/humrep/17.1.44

[20] El-Toukhy, T., Khalaf, Y., Hart, R., Taylor, A. and Braude, P. (2002) Young Age Does Not Protect against the Adverse Effects of Reduced Ovarian Reserve-An Eight Year Study. Human Reproduction, 17, 1519-1524. http://dx.doi.org/10.1093/humrep/17.6.1519

[21] Polyzos, N.P., Blockeel, C., Verpoest, W., De Vos, M., Stoop, D., Vloeberghs, V., Camus, M., Devroey, P. and Tournayé, H. (2012) Live Birth Rates Following Natural Cycle IVF in Women with Poor Ovarian Response According to the Bologna Criteria. Human Reproduction, 27, 3481-3486. http://dx.doi.org/10.1093/humrep/des318

[22] Goverde, A.J., McDonnell, J., Schats, R., Vermeiden, J.P., Homburg, R. and Lambalk, C.B. (2005) Ovarian Response to Standard Gonadotrophin Stimulation for IVF Is Decreased Not Only in Older But Also in Younger Women in Couples with Idiopathic and Male Subfertility. Human Reproduction, 20, 1573-1577. http://dx.doi.org/10.1093/humrep/deh827

[23] Molloy, D., Martin, M., Speirs, A., Lopata, A., Clarke, G., McBain, J., Ngu, A. and Johnston, I.H. (1987) Performance of Patients with a "Frozen Pelvis" in an in Vitro Fertilization Program. Fertility and Sterility, 47, 450-455.

[24] Oehninger, S., Scott, R., Muasher, S.J., Acosta, A.A., Jones Jr., H.W. and Rosenwaks, Z. (1989) Effects of the Severity of Tubo-Ovarian Disease and Previous Tubal Surgery on the Results of in Vitro Fertilization and Embryo Transfer. Fertility and Sterility, 51, 126-130.

[25] Keay, S.D., Barlow, R., Eley, A., Masson, G.M., Anthony, F.W. and Jenkins, J.M. (1998) The Relation between Immunoglobulin G Antibodies to Chlamydia trachomatis and Poor Ovarian Response to Gonadotropin Stimulation before in Vitro Fertilization. Fertility and Sterility, 70, 214-218. http://dx.doi.org/10.1016/S0015-0282(98)00145-9

[26] Brodin, T., Bergh, T., Berglund, L., Hadziosmanovic, N. and Holte, J. (2008) Menstrual Cycle Length Is an AgeIndependent Marker of Female Fertility: Results from 6271 Treatment Cycles of in Vitro Fertilization. Fertility and Sterility, 90, 1656-1661. http://dx.doi.org/10.1016/j.fertnstert.2007.09.036 
Scientific Research Publishing (SCIRP) is one of the largest Open Access journal publishers. It is currently publishing more than 200 open access, online, peer-reviewed journals covering a wide range of academic disciplines. SCIRP serves the worldwide academic communities and contributes to the progress and application of science with its publication.

Other selected journals from SCIRP are listed as below. Submit your manuscript to us via either submit@scirp.org or Online Submission Portal.
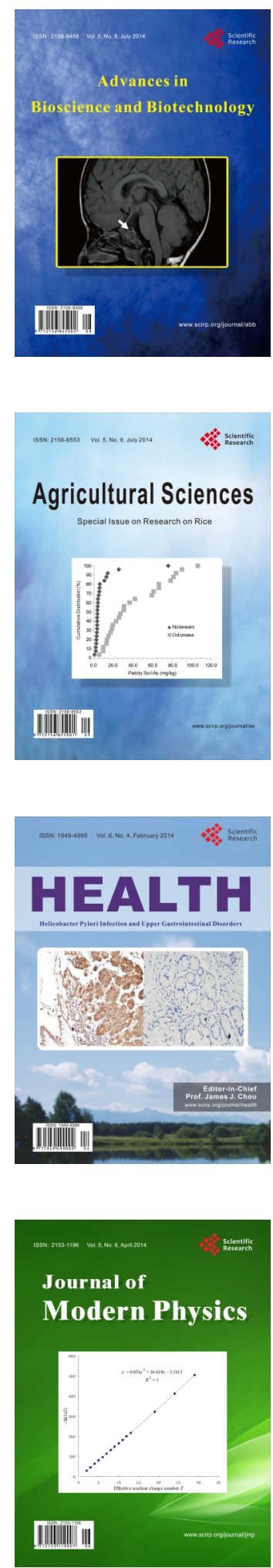
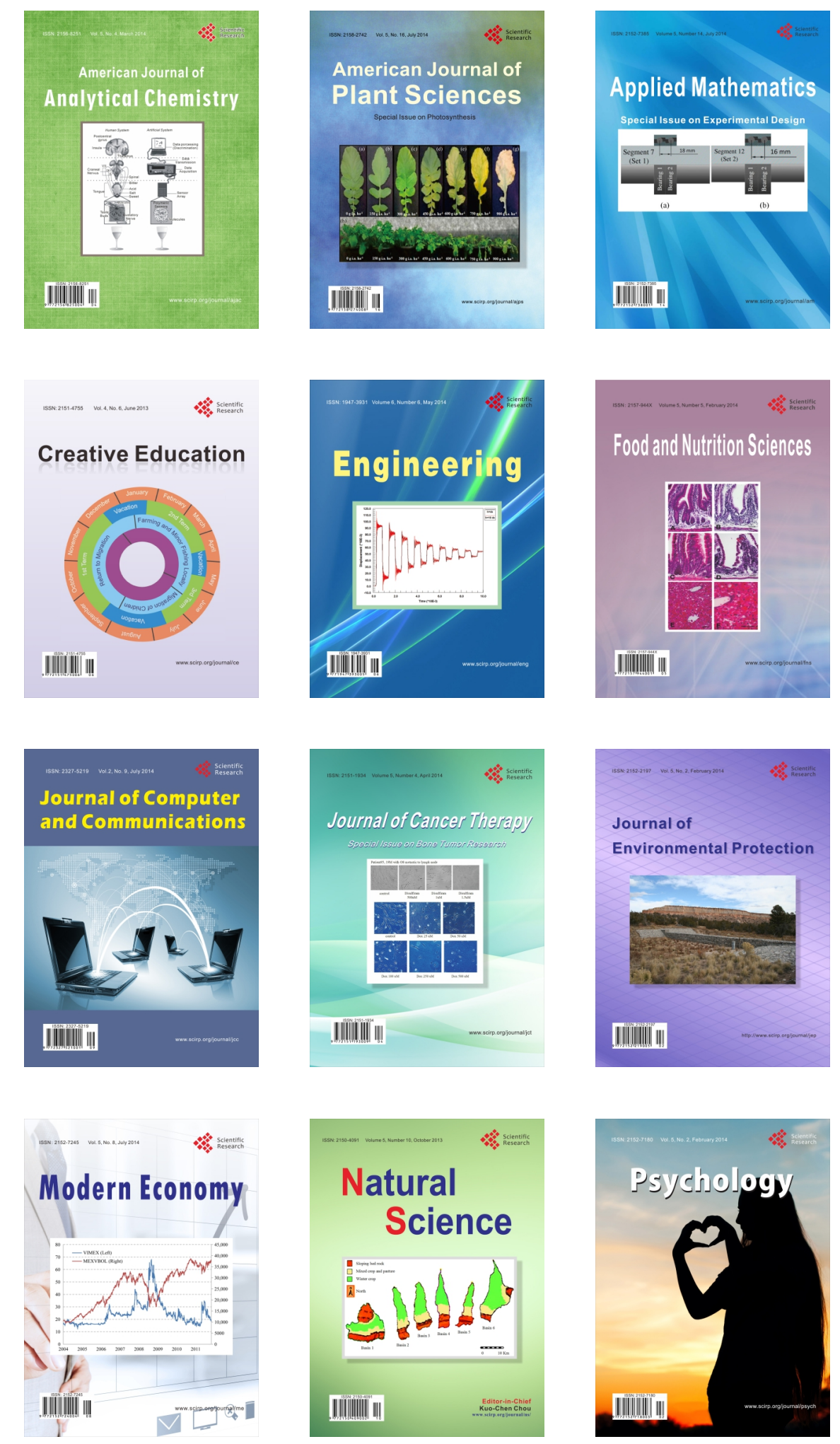